OPEN ACCESS

Edited by:

Giorgio Sandrini

Fondazione Cirna Onlus, Italy

Reviewed by:

Attila Szabo,

University of Oslo, Norway

Olivia Gosseries,

University of Liège, Belgium

Paolo Cardone,

University of Liège, Belgium in collaboration with reviewer $O G$

*Correspondence:

Shariq Mansoor Khan

skhan29@wustl.edu

Specialty section:

This article was submitted to

Neurorehabilitation,

a section of the journal

Frontiers in Neurology

Received: 24 March 2021

Accepted: 29 June 2021

Published: 29 July 2021

Citation:

Khan SM, Carter GT, Aggarwal SK and Holland J (2021) Psychedelics for

Brain Injury: A Mini-Review.

Front. Neurol. 12:685085.

doi: 10.3389/fneur.2021.685085

\section{Psychedelics for Brain Injury: A Mini-Review}

\author{
Shariq Mansoor Khan ${ }^{1 *}$, Gregory T. Carter ${ }^{2}$, Sunil K. Aggarwal ${ }^{3}$ and Julie Holland ${ }^{4}$ \\ ${ }^{1}$ Washington University School of Medicine in St. Louis, St. Louis, MO, United States, ${ }^{2}$ Department of Physical Medicine \& \\ Rehabilitation, St. Luke's Rehabilitation Institute, Spokane, WA, United States, ${ }^{3}$ Advanced Integrative Medical Sciences \\ Institute, Seattle, WA, United States, ${ }^{4}$ Private Practitioner, New York, NY, United States
}

Objective: Stroke and traumatic brain injury (TBI) are among the leading causes of disability. Even after engaging in rehabilitation, nearly half of patients with severe TBI requiring hospitalization are left with major disability. Despite decades of investigation, pharmacologic treatment of brain injury is still a field in its infancy. Recent clinical trials have begun into the use of psychedelic therapeutics for treatment of brain injury. This brief review aims to summarize the current state of the science's relevance to neurorehabilitation, and may act as a resource for those seeking to understand the precedence for these ongoing clinical trials.

Methods: Narrative mini-review of studies published related to psychedelic therapeutics and brain injury.

Results: Recent in vitro, in vivo, and case report studies suggest psychedelic pharmacotherapies may influence the future of brain injury treatment through modulation of neuroinflammation, hippocampal neurogenesis, neuroplasticity, and brain complexity.

Conclusions: Historical data on the safety of some of these substances could serve in effect as phase 0 and phase I studies. Further phase II trials will illuminate how these drugs may treat brain injury, particularly TBI and reperfusion injury from stroke.

Keywords: psychedelics, neuroinflammation, neuroplasticity, stroke, brain injury, review

\section{INTRODUCTION}

Despite millennia of historical use around the world, research into medical uses of psychedelic drugs has been stymied for years by stigma (1). "Classical" psychedelic drugs refer to the most well studied and culturally significant psychedelics, including mescaline, lysergic acid diethylamide (LSD), psilocybin, and dimethyltryptamine (DMT). Though having a wide range of molecular structures and target receptors, psychedelics are unified by their ability to produce marked alterations in sensory perception, consciousness, distortion of time, and perception of reality. Evidence suggests that activation of 5-HT2A receptors (a class of excitatory receptors of serotonin or $5-\mathrm{HT}$ ), is the common mechanism for the psychological experience of classical psychedelics, though these compounds are known to act at other receptors (2).

Currently, however, psychedelics are experiencing a scientific renaissance due to advances in research methodology and changes in the regulation of these substances. Trials of psilocybin for disorders of consciousness, and DMT for stroke, are in discussion to begin in coming years $(3,4)$. In vitro and in vivo studies suggest psychedelics may influence the future of brain injury treatment in both the acute and chronic phases through a variety of mechanisms including modulation of neuroinflammation, neuroplasticity, hippocampal neurogenesis, and increases in brain complexity. 


\section{METHODS}

We conducted a literature search of articles relevant to psychedelic therapeutics for brain injury using PubMed. The search terms are available in the Appendix. Bibliographies of the main review papers were also used to detect other relevant articles. Studies were selected with emphasis to their relevance to psychedelics' purported neuroregenerative and neuroprotective potential, rather than their psychotherapeutic properties. A narrative mini-review format was employed with the intention of providing a brief overview for readers seeking to understand the scientific basis for anticipated clinical trials.

\section{NEUROINFLAMMATION}

Within the brain, depression, addiction, Alzheimer's, and Parkinson's all appear to be linked to neuroinflammatory states (5-7). There are currently three main classes of anti-inflammatory drugs: non-steroidal anti-inflammatory drugs (NSAIDs), steroids such as prednisone, and biologics which act like sponges to "soak up" inflammatory cytokines (1). Psychedelics may represent a fourth class of anti-inflammatory drug.

Neuroinflammation after stroke is responsible for both infarct expansion as well as remodeling and repair $(8,9)$. Modulation of this inflammation is currently a target for new therapies. The inflammatory response to ischemic stroke is thought to derive largely from reperfusion injury (10). In general, there are no conventional medical therapies addressing reperfusion injury after stroke, with the exception of edavarone in Japan, which is only modestly effective (11).

After stroke, immune cells invade the injured tissue, interacting with microglia and neurons (8). Modulating this inflammatory response, particularly through tumor necrosis factor (TNF), interleukin (IL)-1, IL-6, and IL-10, may be the next frontier in stroke recovery $(8,12)$. However, it should be expected that the cytokine response to brain injury has both beneficial and harmful effects to the recovering patient. In contrast to steroids, which cause generalized systemic immunosuppression, psychedelics produce a unique pattern of cytokine expression favoring anti-allergic conditions $(13,14)$. In other words, psychedelics may target many of the pathologic immune responses without exposing the body to the risks of total immune suppression (e.g., serious infection) or potential side effects of existing biologics (e.g., malignancy and cardiovascular disease). Instead, careful regulation of the inflammatory response,

\footnotetext{
Abbreviations: 5HTR, 5-HT receptors; ALS, Amyotrophic lateral sclerosis; DMN, Default-mode network; DMT, N,N-Dimethyltryptamine; DOI, 2,5-dimethoxy-4iodoamphetamine; DOC, Disorder of consciousness; EEG, Electroencephalogram; fMRI, Functional magnetic resonance imaging; IL, Interleukin; LSD, Lysergic acid diethyl amide; LZC, Lempel-Ziv complexity; MAOI, Monoamine oxidase inhibitor; MCAO, Middle cerebral artery occlusion; MDMA, 3,4methylenedioxymethamphetamine; MVF, Mirror visual feedback therapy; NFAT, Nuclear factor of activated T-cells; REM, Rapid eye movement sleep; S1R, Sigma-1 receptor; TBI, Traumatic brain injury; THC, Tetrahydrocannabinol; TLR, Toll-like receptor; TNF, Tumor necrosis factor; SOD, Superoxide dismutase.
}

rather than blunt reduction of the response, or "single-target" approaches, is critical to improved outcomes.

Mizuma and Yenari (10) argue that immunomodulatory therapies have previously not shown efficacy in clinical trials because they largely took place before the use of revascularization techniques. Because reperfusion injury is thought to be the inciting event for much of the neuroinflammatory response, they argue that combining revascularization with immunomodulation may hold promise for stroke treatment. Indeed, Jickling et al.'s (12) review of neutrophil modulation as treatment for stroke notes the contrast seen in the efficacy of these treatments in animal models is dependent on whether reperfusion is induced.

Classical psychedelics act principally on the 5hydroxytryptamine receptors (5-HTRs) to produce their psychological effects, specifically the 5-HT2a receptor (5). These same receptors are well-known to have the potential to regulate inflammation within the central nervous system and peripherally (15). In fact, the 5-HT2a receptor is the most widely expressed serotonin receptor throughout the human body (1). It is present on nearly all tissue and cell types, including all major immune-related cell types (1). However, the highest density of 5-HT2a receptors is found in the brain (1). Though peripheral immunomodulation has been documented with other psychedelics like lysergic acid diethylamide (LSD) (16), 3,4-methylenedioxy-methamphetamine (MDMA) (17), and 2,5-dimethoxy-4-iodoamphetamine (DOI) (13), N,N-Dimethyltryptamine (DMT) has been especially wellstudied with regards to its effects on neuroinflammation and reperfusion injury.

DMT is a psychedelic which is endogenously produced by the human brain, particularly the pineal gland, likely in local concentrations comparable to other monoamine neurotransmitters (18). Though it has been known for at least 40 years that DMT is an endogenously produced hallucinogen, its physiologic function remains elusive. The lack of consensus may be due in part to a paradigm in which the scientific community has assumed DMT can only be, or primarily acts as, a hallucinogen, which keeps research focus on its psychologic effects at 5-HTRs, rather than its non-hallucinogenic effects (9). Recent experiments have shown that an additional receptor, S1R, is critical in the immunomodulating response of DMT.

$\mathrm{S} 1 \mathrm{R}$ dysfunction is known to be involved in a wide range of neurodegenerative diseases including Alzheimer's disease, Parkinson's disease, Huntington's disease, amyotrophic lateral sclerosis (ALS), and TBI (19-21). S1R agonists produce neuroprotective effects via regulation of intracellular calcium, reducing expression of pro-apoptotic genes, and inhibiting antiapoptotic activity by the Bcl-2 gene (22). Specifically, DMT's action at S1Rs has been shown in murine models to modulate inflammation by reducing IL-1b, IL-6, TNFa, and IL-8, while increasing the secretion of the anti-inflammatory cytokine IL10, and by inhibiting activation of Th1 ( $\mathrm{T}$ Helper cell type 1) and Th17 (T Helper cell type 17) subsets (20). In mice, S1R is known to play an important role in the endoplasmic reticulum stress response, including oxidative stress, probably by up-regulating antioxidants, quinine oxidoreductase 1 and superoxide dismutase (SOD) (23). Being present in reactive 
astrocytes, microglia, and neurons, it has also been shown to regulate neuritic outgrowth, myelination, synaptogenesis, and neuro-regeneration $(22,24)$. It should be noted that $\mathrm{S} 1 \mathrm{R}$ is now known to have multiple endogenous agonists, such as progesterone (25). However, with no clear alternative explanation, it may be reasonable to hypothesize that DMT has some, or all, of these neuroprotective and anti-inflammatory physiologic functions as well. The only clinical trial of a selective S1R agonist for treatment of stroke showed statistically significant functional recovery in post-hoc analysis of moderatelyto-severely affected patients (26).

DMT has been shown to reduce ischemic brain injury after middle cerebral artery occlusion (MCAO) through S1R dependent activity in murine models (27). Nardai et al. (27) documented a significant reduction of the infarct core volume in rats at $24 \mathrm{~h}$ post MCAO with administration of DMT, as well as enhanced functional regeneration of the affected limb at 30 days post MCAO (27). These results are consistent with other in vitro studies showing cytoprotective effects of DMT against reperfusion injury (28). Furthermore, similar effects have been documented in human-specific models including human cerebral organoids. Dakic et al. (22) were the first to document that a closely related congener of DMT, 5-MeO-DMT, found in high concentrations of the toxin of Incilius alvarius (Colorado River Toad), favorably altered the cerebral proteome with regard to factors involved with plasticity and neuroprotection including long-term potentiation, apoptosis, morphogenesis/maturation of dendritic spines, and T-lymphocyte differentiation, while inhibiting factors involved in neurodegeneration and cell death. The authors note that other dimethyltryptamines may have similar effects, but their results show that the mechanisms may be different and that each deserves careful study. Previous studies in monolayer neuronal cultures did not show the same effects, suggesting a more complex circuitry is required for these effects (22).

\section{HIPPOCAMPAL NEUROGENESIS}

TBI and stroke alter hippocampal neurogenesis in murine models $(29,30)$. Though hippocampal neurogenesis is recognized as an important component of cognitive recovery from TBI and stroke, there is not a direct correlation between increased neurogenesis and recovery. Complicating factors include the nature of the injury, the timing of intervention, how the cells integrate into the hippocampal circuits, and whether the target of intervention is either increased neuronal proliferation or increased survival. While hippocampal neurogenesis after TBI is implicated in improved cognition, relief from depressed mood, and encoding of episodic memory, it is also associated with pro-epileptogenic changes and spatial memory impairment $(29,31,32)$.

Though many factors are implicated in hippocampal neurogenesis, one of the most important is 5HTR stimulation $(33,34)$. Acute administration of psilocybin to mice alters hippocampal neurogenesis in a non-linear fashion (35). Low doses lead to increased neurogenesis while higher doses inhibit it. However, increased neurogenesis has also been seen when high dose psilocybin was administered onceper-week, avoiding the issue of rapid tolerance buildup via 5HTR downregulation (33). Targeting hippocampal neurogenesis for treatment of brain injury and other psychiatric and neurologic disorders is an emerging area of research (36).

\section{NEUROPLASTICITY}

Novel interventional approaches hold promise to improve functional outcomes after brain injury by inducing neural plasticity. However, like targeting inflammation and hippocampal neurogenesis, targeting neuroplasticity is a double-edged sword. Ischemia induced plasticity may be responsible for recovery of function, but also drive complications such as epilepsy and memory disturbance. A full discussion on ischemia induced plasticity is available elsewhere (37).

Functional recovery due to post-stroke plasticity is currently most effectively recruited through intensive physical therapy (37). However, both non-invasive techniques for inducing neuroplasticity such as transcranial magnetic stimulation and direct current stimulation, as well as invasive techniques such as deep brain stimulation, show promising results for stroke recovery (37).

The search for pharmacologic agents which stimulate neuroplasticity after brain injury, including amphetamines, dopaminergic, serotonergic, noradrenergic, and cholinergic agents, has thus far been inconclusive. To our knowledge, the effect of psychedelics on neuroplasticity has not been tested in brains subjected to injury such as stroke. However, recent reports demonstrate that psychedelics promote both structural and functional neuroplasticity in non-injured brains. The persistent symptom improvement in psychiatric disorders with administration of psychedelics has been proposed to be driven by this neuroplastic adaptation (2). Ly et al. (38) found that some psychedelics were more efficacious (e.g., MDMA) or more potent (e.g., LSD) than ketamine in promoting plasticity. These results were demonstrated in vivo in both non-human vertebrates and invertebrates, suggesting that these mechanisms are evolutionarily conserved.

One hypothesized mechanism for psychedelics' beneficial effect on neuroplasticity in brain injury is via Brain-derived neurotrophic factor (BDNF), which is well-known to be implicated in both neuritogenesis and spinogenesis (39). In Ly et al.'s (38) experiments, they found that many psychedelics rivaled administration of pure BDNF in their ability to induce plasticity. At least one randomized control trial has demonstrated increases in serum BDNF in volunteers with administration of a DMT containing tisane, ayahuasca, which persisted for $48 \mathrm{~h}$ (40). Importantly, ayahuasca, a traditional South American ceremonial brew, contains not only DMT, but also $\beta$-carbolines which act as reversible monoamine oxidase inhibitors (MAOIs) potentiating and prolonging the effects of DMT (40). However, it should be noted that many other non-psychedelic drugs have failed to alter the course of brain injury through induction of BDNF. 
However, there is also evidence to suggest that S1R is an important part of the plasticity response to stroke. For example, Ruscher et al. (41) found that delivery of a synthetic S1R agonist enhanced plasticity-mediated recovery of lost sensorimotor function in rats, even when initiation of therapy was started up to 2 days after MCAO, possibly expanding the currently very narrow therapeutic window for acute stroke.

Mirror visual-feedback (MVF) has been shown to enhance key features of neuroplasticity including cross-modal cortical reorganization and learning (42). In patients $<12$ months post stroke, MVF therapy enhances functional recovery of lower limbs and hands $(43,44)$. Psilocybin combined with mirror visual-feedback has been shown in a case report to have a dose-dependent reduction in phantom limb associated pain, hypothesized to be due to facilitating MVF's ability to "unlearn" paralysis via 5HTR-dependent changes in neuroplasticity (42). Clinical trials for use of psilocybin in phantom limb pain are currently underway (45).

\section{INCREASE IN BRAIN COMPLEXITY}

Disorders of consciousness (DOC) can arise from a variety of brain injuries including trauma, hypoglycemia, anoxia, and stroke. Between 4 and $38 \%$ of stroke patients will experience a DOC (46). Though many therapies have been proposed for patients with DOC, including pharmacologic (e.g., amantadine, D-amphetamine, levodopa, modafinil, and zolpidem), invasive and non-invasive stimulation (e.g., transcranial direct current stimulation), benefits for functional recovery are usually modest and current evidence supporting their use is inconsistent (47-50). Other stimulants acting on dopamine including apomorphine may hold promise (51).

Scott and Carhart-Harris have proposed an experimental protocol for testing the capacity of psilocybin to increase conscious awareness in patients with DOC (52). They hypothesize psychedelics increase brain activity complexity and conscious content, in contrast to current stimulant drugs that increase arousal. However, to our knowledge there are no studies of brain complexity measures in DOC patients given stimulants. Their hypothesis relies on findings that psilocybin increases brain complexity, and that these particular measures of complexity, namely perturbational complexity index (53) and the closely related Lempel-Ziv complexity (LZC), reliably predict conscious level. They argue that psychedelic-related elevations in LZC reflect an increased richness of conscious experience, and that targeting increases in conscious content, rather than arousal, as with stimulants, may be key to increasing conscious awareness in DOC patients.

In human subjects, increases in LZC have been observed in excess of those seen in normal wakefulness with administration of psilocybin, LSD, and ketamine at psychedelic doses (54). This increase in complexity has also been demonstrated via other measures including electroencephalogram (EEG) and functional magnetic resonance imaging (fMRI) (55).

Psychedelics are thought to increase brain complexity primarily through 5HTRs (53). As previously discussed, 5HTR agonism is associated with increased neuroplasticity, while antagonism is associated with reduced cognitive flexibility and increased slow-wave sleep and sedation $(56,57)$. These receptors are most densely expressed in the cortical areas belonging to the default-mode network (DMN) (52). The DMN is known to be implicated in conscious processing as well as the subjective experience of psychedelic states (52). Strength of DMN connectivity is significantly decreased in stroke patients with DOCs, and is highly correlated with Glasgow Coma Scale scores (58). Secondarily, 5HTRs have been shown to play an important role in the control of thalamo-frontal connectivity, known to be important for consciousness $(59,60)$.

\section{CONCLUSIONS}

Psychedelics may play a future role in treatment of brain injury through a variety of mechanisms. Though these are a novel class of drugs deserving close study, more data are necessary to prove their efficacy for treatment of brain injury, as historically many compounds have seemed promising in vitro, including likely hundreds of compounds thought to facilitate neuroplasticity and neuroprotection, but have not borne out in clinical trials $(61,62)$. There is already mixed evidence to suggest the use of non-classical psychedelics ketamine $(63,64)$, as well as tetrahydrocannabinol (THC) $(65,66)$ and cannabidiol $(67,68)$, as neuroprotectants after TBI and stroke. Presence of THC on urine drug screen is associated with decreased mortality in adult patients sustaining TBI (65). However, randomized control trials of a nonpsychoactive cannabinoid analog, dexanabinol, administered once after TBI has failed to show benefit over placebo in increasing Glasgow Coma Scores at 6 months post-injury (69). One explanation for this discrepancy may be the lack of "entourage" effect whereby various cannabinoids and cannabis phytochemicals are clinically more efficacious when working synergistically compared to administration of a single cannabinoid in isolation (70), though the existence of such an effect with cannabis is contentious with mixed evidence for its existence (71). Of note, an "entourage" effect has been demonstrated with psychedelic mushrooms in which whole mushroom extracts are on the order of 10 times more potent than purified psilocin administered alone in neurobehavioral rat models (72).

Another possible explanation is that the subjective hallucinogenic effects are necessary for some, or all, of psychedelics' therapeutic effects. This remains a fundamental research question and strong evidence exists on both sides of the debate (73). Few human trials on the therapeutic effects of psychedelics at sub-behavioral "micro-" doses have been completed, but results from animal and cell studies for their use as anti-inflammatories are promising (5). In contrast, studies on the psychological effects of micro-dosing psychedelics seem to be explained by placebo effect $(74,75)$. Extrapolating from animal studies, the doses required at least for the anti-inflammatory effects of psychedelics are predicted to be magnitudes less than threshold for hallucinations (13). 
Furthermore, recent studies treating rat models of asthma with 2,5-dimethoxyphenethylamine $(2 \mathrm{C}-\mathrm{H})$ which is a nonhallucinogenic 5-HT2a agonist structurally related to MDMA, suggest that psychedelics' behavioral and anti-inflammatory effects may have separate, but related, underlying mechanisms (76). A non-hallucinogenic analog of ibogaine has been shown to induce structural neuroplasticity, as well as reduce depression, alcohol-, and heroin-seeking behavior in rodents, similarly to its hallucinogenic variant (77). In contrast, Brouwer and Carhart-Harris have introduced a construct known as the "pivotal mental state" to explain the evolutionary function that 5-HT2a receptor agonism has in inducing neurologic hyper-plasticity and psychological adaptation when the body is exposed to subjective acute stress (e.g., hallucinations, intense spiritual experience, psychosis, trauma) (78). They hypothesize that "psychedelics hijack a system that has evolved to mediate rapid and deep learning" to provide a psychological "fresh start" or "rebirth." It is likely that some, but not all, of the psychological therapeutic benefits of psychedelics is dependent on the subjective experience (73), but whether the same can be said for their use in brain injury is unclear.

Classical psychedelics have millennia of historical use, do not have significant risk of dependence, and are safe to use under close medical supervision $(79,80)$. Though there should be caution in over-interpreting the relevance of the aforementioned animal studies' relevance to human pathological states, this historical data should serve in effect as phase 0 and phase I studies (9). However, presumably much of this historical data is based on intermittent, infrequent dosing, so trial safety data may need to be repeated with continuous, regular doses. Further phase II

\section{REFERENCES}

1. Nichols DE, Johnson MW, Nichols CD. Psychedelics as medicines: an emerging new paradigm. Clin Pharmacol Ther. (2017) 101:20919. doi: $10.1002 / \mathrm{cpt} .557$

2. Vollenweider FX, Preller KH. Psychedelic drugs: neurobiology and potential for treatment of psychiatric disorders. Nat Rev Neurosci. (2020) 21:61124. doi: 10.1038/s41583-020-0367-2

3. DMT- Stroke Program. Algernon Pharmaceuticals. (2021). Available online at: https://algernonpharmaceuticals.com/dmt-stroke-program/ (accessed May 19, 2021).

4. Gosseries O, Martial C. The use of psychedelics in the treatment of disorders of consciousness. ALIUS BULLETIN: Exploring the Diversity of Consciousness (no 4) (2021). Available online at: https://orbi.uliege.be/handle/2268/254726

5. Flanagan TW, Nichols CD. Psychedelics as anti-inflammatory agents. Int Rev Psychiatry. (2018) 30:363-75. doi: 10.1080/09540261.2018.1481827

6. Caggiu E, Arru G, Hosseini S, Niegowski M, Sechi G, Zarbo I, et al. Inflammation, infectious triggers, and Parkinson's disease. Front Neurol. (2019) 10:122. doi: 10.3389/fneur.2019.00122

7. Szabo A, Frecska E. Dimethyltryptamine (DMT): a biochemical Swiss Army knife in neuroinflammation and neuroprotection? Neural Regen Res. (2016) 11:396-7. doi: 10.4103/1673-5374.179041

8. Lambertsen KL, Finsen B, Clausen BH. Post-stroke inflammationtarget or tool for therapy? Acta Neuropathol. (2019) 137:693714. doi: 10.1007/s00401-018-1930-z

9. Winkelman MJ, Sessa B. Advances in Psychedelic Medicine: State-of-the-Art Therapeutic Applications. Santa Barbara, CA: ABC-CLIO (2019). trials will illuminate how these drugs may treat brain injury, particularly TBI and reperfusion injury from stroke.

Further study and design of non-psychoactive analogs may answer fundamental questions regarding the interplay of hallucinations with other properties of psychedelic therapy, as well as facilitate more practical use in acute hospital settings. The subjective hallucinogenic effects of psychedelics, with clinical psychological support, may also prove valuable in fostering recovery from brain injury from a trauma recovery rehabilitation psychology standpoint due to their anti-PTSD and anti-depression effects that are now well-documented (81, 82). Depression and PTSD significantly hinder recovery from stroke and TBI $(83,84)$. Psychedelics' use in this area may be more immediately clinically relevant in this area than in the neuroprotection and neuro-regeneration, where comparatively less is known. Further research is needed to determine the optimal timing after injury and route of administration for neuroprotective effects. All the compounds discussed in this paper have reasonable oral absorption. Route of administration would impact the degree and timeliness of the pharmacological effects yet there are not enough studies specifically on this aspect to make recommendations. The emerging use of intranasal route of administration may afford rapid induction into the central nervous system and could be a promising future option (85).

\section{AUTHOR CONTRIBUTIONS}

SK and GC contributed to conception and design of the study. SK wrote the first draft of the manuscript. SK, GC, SA, and JH wrote sections of the manuscript. All authors contributed to manuscript revision, read, and approved the submitted version.

10. Mizuma A, Yenari MA. Anti-inflammatory targets for the treatment of reperfusion injury in stroke. Fron Neurol. (2017) 8:467. doi: 10.3389/fneur.2017.00467

11. Susumu K, Shingo F, Tatsuyoshi I, Shunichi F, Shotai K. Effect of edaravone on neurological symptoms in real-world patients with acute ischemic stroke. Stroke. (2019) 50:1805-11. doi: 10.1161/STROKEAHA.118.0 24351

12. Jickling GC, Liu D, Ander BP, Stamova B, Zhan X, Sharp FR. Targeting neutrophils in ischemic stroke: translational insights from experimental studies. J Cereb Blood Flow Metab. (2015) 35:888-901. doi: 10.1038/jcbfm.2015.45

13. Nau F, Yu B, Martin D, Nichols CD. Serotonin 5-HT2A receptor activation blocks TNF- $\alpha$ mediated inflammation in vivo. PLoS ONE. (2013) 8:e75426. doi: 10.1371/journal.pone.0075426

14. Thompson C, Szabo A. Psychedelics as a novel approach to treating autoimmune conditions. Immunol Lett. (2020) 228:4554. doi: 10.1016/j.imlet.2020.10.001

15. Shajib MS, Khan WI. The role of serotonin and its receptors in activation of immune responses and inflammation. Acta Physiol. (2015) 213:56174. doi: 10.1111/apha. 12430

16. House RV, Thomas PT, Bhargava HN. Immunological consequences of in vitro exposure to lysergic acid diethylamide (LSD). Immunopharmacol Immunotoxicol. (1994) 16:23-40. doi: 10.3109/089239794090 29898

17. Boyle NT, Connor TJ. Methylenedioxymethamphetamine ('Ecstasy')-induced immunosuppression: a cause for concern? Br J Pharmacol. (2010) 161:1732. doi: 10.1111/j.1476-5381.2010.00899.x 
18. Dean JG, Liu T, Huff S, Sheler B, Barker S, Strassman R, et al. Biosynthesis and extracellular concentrations of N,Ndimethyltryptamine (DMT) in mammalian brain. Sci Rep. (2019) 9:1-11. doi: 10.1038/s41598-019-45812-w

19. Nguyen L, Lucke-Wold BP, Mookerjee SA, Cavendish J, Robson M, Scandinaro A, et al. Role of sigma-1 receptors in neurodegenerative diseases. J Pharmacol Sci. (2015) 127:17-29. doi: 10.1016/j.jphs.2014.12.005

20. Szabo A, Kovacs A, Frecska E, Rajnavolgyi E. Psychedelic N,Ndimethyltryptamine and 5- methoxy-N,N-dimethyltryptamine modulate innate and adaptive inflammatory responses through the sigma-1 receptor of human monocyte-derived dendritic cells. PLoS ONE. (2014) 9:e106533. doi: 10.1371/journal.pone.0106533

21. Jia J, Cheng J, Wang C, Zhen X. Sigma-1 receptor-modulated neuroinflammation in neurological diseases. Front Cell Neurosci. (2018) 12:314. doi: 10.3389 /fncel.2018.00314

22. Dakic V, Minardi Nascimento J, Costa Sartore R, Maciel R, de Araujo D, Ribeiro S, et al. Short term changes in the proteome of human cerebral organoids induced by 5-MeO-DMT. Sci Rep. (2017) 7:12863. doi: 10.1038/s41598-017-12779-5

23. Pal A, Fontanilla D, Gopalakrishnan A, Chae Y-K, Markley JL, Ruoho AE. The sigma-1 receptor protects against cellular oxidative stress and activates antioxidant response elements. Eur J Pharmacol. (2012) 682:1220. doi: 10.1016/j.ejphar.2012.01.030

24. Moritz C, Berardi F, Abate C, Peri F. Live imaging reveals a new role for the sigma-1 $(\sigma 1)$ receptor in allowing microglia to leave brain injuries. Neurosci Lett. (2015) 591:13-8. doi: 10.1016/j.neulet.2015.02.004

25. Fontanilla D, Johannessen M, Hajipour AR, Cozzi NV, Jackson $\mathrm{MB}$, Ruoho AE. The hallucinogen N,N-dimethyltryptamine (DMT) is an endogenous sigma-1 receptor regulator. Science. (2009) 323:934-7. doi: 10.1126/science.1166127

26. Urfer R, Moebius HJ, Skoloudik D, Santamarina E, Sato W, Mita S, et al. Phase II trial of the sigma-1 receptor agonist cutamesine (SA4503) for recovery enhancement after acute ischemic stroke. Stroke. (2014) 45:330410. doi: 10.1161/STROKEAHA.114.005835

27. Nardai S, László M, Szabó A, Alpár A, Hanics J, Zahola P, et al. N,Ndimethyltryptamine reduces infarct size and improves functional recovery following transient focal brain ischemia in rats. Exp Neurol. (2020) 327:113245. doi: 10.1016/j.expneurol.2020.113245

28. Nemes B, Peto K, Németh N, Mester A, Magyar Z, Ghanem S, et al. N,Ndimethyltryptamine prevents renal ischemiareperfusion injury in a rat model. Transplant Proc. (2019) 51:1268-75. doi: 10.1016/j.transproceed.2019.04.005

29. Woitke F, Ceanga M, Rudolph M, Niv F, Witte O, Redecker C, et al. Adult hippocampal neurogenesis poststroke: more new granule cells but aberrant morphology and impaired spatial memory. PLOS ONE. (2017) 12:e0183463. doi: 10.1371/journal.pone.0183463

30. Redell JB, Maynard ME, Underwood EL, Vita SM, Dash PK, Kobori N. Traumatic brain injury and hippocampal neurogenesis: functional implications. Exp Neurol. (2020) 331:113372. doi: 10.1016/j.expneurol.2020.113372

31. Ngwenya LB, Danzer SC. Impact of traumatic brain injury on neurogenesis. Front Neurosci. (2019) 12:1014. doi: 10.3389/fnins.2018. 01014

32. Shors TJ, Miesegaes G, Beylin A, Zhao M, Rydel T, Gould E. Neurogenesis in the adult is involved in the formation of trace memories. Nature. (2001) 410:372-6. doi: 10.1038/35066584

33. Catlow BJ, Jalloh A, Sanchez-Ramos J. Chapter 77 - hippocampal neurogenesis: effects of psychedelic drugs. In: Preedy VR, editor. Neuropathology of Drug Addictions and Substance Misuse. London: Academic Press (2016). p. 821-31.

34. Malberg JE, Eisch AJ, Nestler EJ, Duman RS. Chronic antidepressant treatment increases neurogenesis in adult rat hippocampus. $J$ Neurosci. (2000) 20:9104-10. doi: 10.1523/JNEUROSCI.20-24-0910 4.2000

35. Catlow BJ, Song S, Paredes DA, Kirstein CL, Sanchez-Ramos J. Effects of psilocybin on hippocampal neurogenesis and extinction of trace fear conditioning. Exp Brain Res. (2013) 228:481-91. doi: 10.1007/s00221-013-3579-0

36. Dhikav V, Anand KS. Is hippocampal atrophy a future drug target? Med Hypotheses. (2007) 68:1300-6. doi: 10.1016/j.mehy.2006.09.040
37. Chen H, Epstein J, Stern E. Neural plasticity after acquired brain injury: evidence from functional neuroimaging. PMßR. (2010) 2:S30612. doi: 10.1016/j.pmrj.2010.10.006

38. Ly C, Greb AC, Cameron LP, Wong J, Barragan E, Wilson P, et al. Psychedelics promote structural and functional neural plasticity. Cell Rep. (2018) 23:317082. doi: 10.1016/j.celrep.2018.05.022

39. Cohen-Cory S, Kidane AH, Shirkey NJ, Marshak S. Brain-derived neurotrophic factor and the development of structural neuronal connectivity. Dev Neurobiol. (2010) 70:271-88. doi: 10.1002/dneu.20774

40. Almeida RN de, Galvão AC de M, da Silva FS, Silva E, Palhano-Fontes F, Maiade-Olveira J, et al. Modulation of serum brain-derived neurotrophic factor by a single dose of ayahuasca: observation from a randomized controlled trial. Front Psychol. (2019) 10:1234. doi: 10.3389/fpsyg.2019.01234

41. Ruscher K, Shamloo M, Rickhag M, Ladunga I, Soriano L, Gisselson L, et al. The sigma-1 receptor enhances brain plasticity and functional recovery after experimental stroke. Brain. (2011) 134:732-46. doi: 10.1093/brain/awq367

42. Ramachandran V, Chunharas C, Marcus Z, Furnish T, Lin A. Relief from intractable phantom pain by combining psilocybin and mirror visual-feedback (MVF). Neurocase. (2018) 24:10510. doi: 10.1080/13554794.2018.1468469

43. Sütbeyaz S, Yavuzer G, Sezer N, Koseoglu BF. Mirror therapy enhances lower-extremity motor recovery and motor functioning after stroke: a randomized controlled trial. Arch Phys Med Rehabil. (2007) 88:5559. doi: 10.1016/j.apmr.2007.02.034

44. Yavuzer G, Selles R, Sezer N, Sütbeyaz S, Bussmann J, Köseoglu F, et al. Mirror therapy improves hand function in subacute stroke: a randomized controlled trial. Arch Phys Med Rehabil. (2008) 89:3938. doi: 10.1016/j.apmr.2007.08.162

45. New Grant Funds Clinical Trial to Assess Psychedelic as Treatment for Phantom Limb Pain. Available online at: https://ucsdnews.ucsd.edu/pressrelease/newgrant-funds-clinical-trial-to-assess-psychedelic-as-treatment-for-phantomlimb-pain (accessed May 23, 2021).

46. Li J, Wang D, Tao W, Dong W, Zhang J, Yang J, et al. Early consciousness disorder in acute ischemic stroke: incidence, risk factors and outcome. BMC Neurol. (2016) 16:140. doi: 10.1186/s12883-016-0666-4

47. Mura E, Pistoia F, Sara M, Sacco S, Carolei A, Govoni S. Pharmacological modulation of the state of awareness in patients with disorders of consciousness: an overview. Curr Pharm Des. (2014) 20:4121-39. doi: 10.2174/13816128113196660658

48. Giacino JT, Whyte J, Bagiella E, Kalmar K, Childs N, Khademi A, et al. Placebo-controlled trial of amantadine for severe traumatic brain injury. $N$ Engl J Med. (2012) 366:819-26. doi: 10.1056/NEJMoa1102609

49. Bomalaski MN, Claflin ES, Townsend W, Peterson MD. Zolpidem for the treatment of neurologic disorders: a systematic review. JAMA Neurol. (2017) 74:1130-9. doi: 10.1001/jamaneurol.2017.1133

50. Aloi D, della Rocchetta AI, Ditchfield A, Coulborn S, Fernández-Espejo D. Therapeutic use of transcranial direct current stimulation in the rehabilitation of prolonged disorders of consciousness. Front Neurol. (2021) 12:632572. doi: 10.3389/fneur.2021.632572

51. Sanz LRD, Lejeune N, Blandiaux S, Bonin E, Thibaut A, Stender J, et al. Treating disorders of consciousness with apomorphine: protocol for a double-blind randomized controlled trial using multimodal assessments. Front Neurol. (2019) 10:248. doi: 10.3389/fneur.2019.00248

52. Scott G, Carhart-Harris RL. Psychedelics as a treatment for disorders of consciousness. Neurosci Conscious. (2019) 2019. doi: 10.1093/nc/niz003

53. Casali AG, Gosseries O, Rosanova M, Boly M, Sarasso S, Casali $\mathrm{K}$, et al. A theoretically based index of consciousness independent of sensory processing and behavior. Sci Transl Med. (2013) 5:198ra105. doi: 10.1126/scitranslmed.3006294

54. Schartner MM, Carhart-Harris RL, Barrett AB, Seth $A K$, Muthukumaraswamy SD. Increased spontaneous MEG signal diversity for psychoactive doses of ketamine, LSD and psilocybin. Sci Rep. (2017) 7:46421. doi: 10.1038/srep46421

55. Carhart-Harris RL. The entropic brain - revisited. Neuropharmacology. (2018) 142:167-78. doi: 10.1016/j.neuropharm.2018.03.010

56. Carhart-Harris RL, Nutt DJ. Serotonin and brain function: a tale of two receptors. J Psychopharmacol. (2017) 31:1091120. doi: $10.1177 / 0269881117725915$ 
57. Vanover KE, Davis RE. Role of 5-HT2A receptor antagonists in the treatment of insomnia. Nat Sci Sleep. (2010) 2:139. doi: 10.2147/NSS.S6849

58. Tsai Y-H, Yuan R, Huang Y-C, Yeh M-Y, Lin C-P, Biswal BB. Disruption of brain connectivity in acute stroke patients with early impairment in consciousness. Front Psychol. (2014) 4:956. doi: 10.3389/fpsyg.2013. 00956

59. Barre A, Berthoux C, De Bundel D, Valjent E, Bockaert J, Marin P, et al. Presynaptic serotonin 2A receptors modulate thalamocortical plasticity and associative learning. Proc Natl Acad Sci USA. (2016) 113:E138291. doi: 10.1073/pnas.1525586113

60. Giacino JT, Fins JJ, Laureys S, Schiff ND. Disorders of consciousness after acquired brain injury: the state of the science. Nat Rev Neurol. (2014) 10:99114. doi: 10.1038/nrneurol.2013.279

61. Diaz-Arrastia R, Kochanek PM, Bergold P, Kenney K, Marx C, Grimes J, et al. Pharmacotherapy of traumatic brain injury: state of the science and the road forward: report of the department of defense neurotrauma pharmacology workgroup. J Neurotrauma. (2014) 31:135-58. doi: 10.1089/neu.2013.3019

62. O'Collins VE, Macleod MR, Donnan GA, Horky LL, van der Worp BH, Howells DW. 1,026 experimental treatments in acute stroke. Ann Neurol. (2006) 59:467-77. doi: 10.1002/ana.20741

63. Godoy DA, Badenes R, Pelosi P, Robba C. Ketamine in acute phase of severe traumatic brain injury "an old drug for new uses?" Critical Care. (2021) 25:1-7. doi: 10.1186/s13054-020-03452-x

64. Xiong Z, Chang L, Qu Y, Pu Y, Wang S, Fujita Y, et al. Neuronal brain injury after cerebral ischemic stroke is ameliorated after subsequent administration of (R)-ketamine, but not (S)-ketamine. Pharmacol Biochem Behav. (2020) 191:172904. doi: 10.1016/j.pbb.2020.172904

65. Nguyen BM, Kim D, Bricker S, Bongard F, Neville A, Putnam B, et al. Effect of marijuana use on outcomes in traumatic brain injury. Am Surg. (2014) 80:979-83. doi: 10.1177/000313481408001015

66. Elias D, Plurad D, Bender M. Relationship of time of injury marijuana exposure and traumatic brain injury: a systematic review. J Trauma Nursing. (2020) 27:360-8. doi: 10.1097/JTN.0000000000000544

67. Choi S-H, Mou Y, Silva AC. Cannabis and cannabinoid biology in stroke. Stroke. (2019) 50:2640-5. doi: 10.1161/STROKEAHA.118.023587

68. Grenier K, Ponnambalam F, Lee D, Lauwers R, Bhalerao S. Cannabis in the treatment of traumatic brain injury: a primer for clinicians. Can J Neurol Sci. (2020) 47:11-7. doi: 10.1017/cjn.2019.298

69. Maas AI, Murray G, Henney III H, Kassem N, Legrand V, Mangelus M, et al. Efficacy and safety of dexanabinol in severe traumatic brain injury: results of a phase III randomised, placebo-controlled, clinical trial. Lancet Neurol. (2006) 5:38-45. doi: 10.1016/S1474-4422(05)70253-2

70. Blasco-Benito S, Seijo-Vila M, Caro-Villalobos M, Tundidor I, Andradas C, García-Taboada E, et al. Appraising the "entourage effect": antitumor action of a pure cannabinoid versus a botanical drug preparation in preclinical models of breast cancer. Biochem Pharmacol. (2018) 157:28593. doi: 10.1016/j.bcp.2018.06.025

71. Bonn-Miller MO, ElSohly MA, Loflin MJE, Chandra S, Vandrey R. Cannabis and cannabinoid drug development: evaluating botanical versus single molecule approaches. Int Rev Psychiatry. (2018) 30:277-84. doi: 10.1080/09540261.2018.1474730

72. Zhuk O, Jasicka-Misiak I, Poliwoda A, Kazakova A, Godovan V, Halama M, et al. Research on acute toxicity and the behavioral effects of methanolic extract from psilocybin mushrooms and psilocin in mice. Toxins. (2015) 7:1018-29. doi: 10.3390/toxins7041018

73. Majić T, Schmidt TT, Gallinat J. Peak experiences and the afterglow phenomenon: when and how do therapeutic effects of hallucinogens depend on psychedelic experiences? J Psychopharmacol. (2015) 29:24153. doi: $10.1177 / 0269881114568040$
74. van Elk M, Fejer G, Lempe P, Prochazckova L, Kuchar M, Hajkova K, et al. Effects of psilocybin microdosing on awe and aesthetic experiences: a preregistered field and lab-based study. Psychopharmacology. (2021) 1-16. doi: 10.1007/s00213-021-05857-0. [Epub ahead of print].

75. Szigeti B, Kartner L, Blemings A, Rosas F, Feilding A, Nutt D, et al. Selfblinding citizen science to explore psychedelic microdosing. Elife. (2021) 10:e62878. doi: 10.7554/eLife.62878

76. Flanagan TW, Billac GB, Landry AN, Sebastian MN, Cormier SA, Nichols CD. Structure-activity relationship analysis of psychedelics in a rat model of asthma reveals the anti-inflammatory pharmacophore. ACS Pharmacol Transl Sci. (2020). doi: 10.1021/acsptsci.0c00063

77. Cameron LP, Tombari RJ, Lu J, Pell A, Hurley Z, Ehinger Y, et al. A non-hallucinogenic psychedelic analogue with therapeutic potential. Nature. (2021) 589:474-9. doi: 10.1038/s41586-020-3008-Z

78. Brouwer A, Carhart-Harris RL. Pivotal mental states. J Psychopharmacol. (2021) 35:319-52. doi: 10.1177/0269881120959637

79. Davis AK, Barsuglia JP, Lancelotta R, Grant RM, Renn E. The epidemiology of 5- methoxy- N, N-dimethyltryptamine (5-MeO-DMT) use: benefits, consequences, patterns of use, subjective effects, and reasons for consumption. J Psychopharmacol. (2018) 32:779-92. doi: 10.1177/0269881118769063

80. Johnson MW, Griffiths RR. Potential therapeutic effects of psilocybin. Neurotherapeutics. (2017) 14:734-40. doi: 10.1007/s13311-0170542-y

81. Davis AK, Barrett FS, May DG, Cosimano M, Sepeda N, Johnson $M$, et al. Effects of psilocybin-assisted therapy on major depressive disorder: a randomized clinical trial. JAMA Psychiatry. (2021) 78:4819. doi: 10.1001/jamapsychiatry.2020.3285

82. Carhart-Harris R, Giribaldi B, Watts R, Baker-Jones M, Murphy-Biener A, Murphy R, et al. Trial of psilocybin versus escitalopram for depression. N Engl J Med. (2021) 384:1402-11. doi: 10.1056/NEJMoa2032994

83. Lavoie S, Sechrist S, Quach N, Ehsanian R, Duong T, Gotlib I, et al. Depression in men and women one year following traumatic brain injury (TBI): a tbi model systems study. Front Psychol. (2017) 8:634. doi: 10.3389/fpsyg.2017.00634

84. Gilbert KS, Kark SM, Gehrman P, Bogdanova Y. Sleep disturbances, TBI and PTSD: implications for treatment and recovery. Clin Psychol Rev. (2015) 40:195-212. doi: 10.1016/j.cpr.2015.05.008

85. Cassano R, Servidio C, Trombino S. Biomaterials for drugs nose-brain transport: a new therapeutic approach for neurological diseases. Materials. (2021) 14:1802. doi: 10.3390/ma14071802

Conflict of Interest: The authors declare that the research was conducted in the absence of any commercial or financial relationships that could be construed as a potential conflict of interest.

Publisher's Note: All claims expressed in this article are solely those of the authors and do not necessarily represent those of their affiliated organizations, or those of the publisher, the editors and the reviewers. Any product that may be evaluated in this article, or claim that may be made by its manufacturer, is not guaranteed or endorsed by the publisher.

Copyright (c) 2021 Khan, Carter, Aggarwal and Holland. This is an open-access article distributed under the terms of the Creative Commons Attribution License (CC $B Y)$. The use, distribution or reproduction in other forums is permitted, provided the original author(s) and the copyright owner(s) are credited and that the original publication in this journal is cited, in accordance with accepted academic practice. No use, distribution or reproduction is permitted which does not comply with these terms. 


\section{APPENDIX}

((psychedelics) OR (psychedelic) or (psilocybin) or (psilocin) or (dimethyltryptamine) or (N,N-Dimethyltryptamine) or $(\mathrm{N}, \mathrm{N}-\mathrm{DMT})$ or $(5-\mathrm{MeO}-\mathrm{DMT})$ or (MDMA) or $(3,4-$ Methylenedioxymethamphetamine) or (LSD) or (lysergic acid diethylamide) ) and ((disorders of consciousness) or (DOC) or (inflammation) or (stroke) or (brain injury) or (reperfusion) or (TBI) or (trauma) or (neuroplasticity)). 